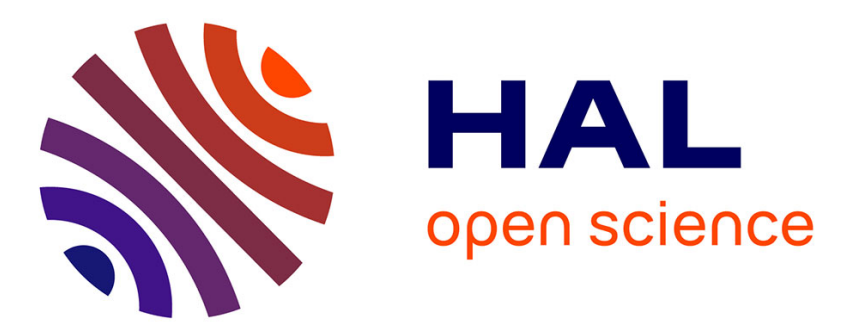

\title{
Flow characteristics around a plate withdrawn from a bath of yield stress fluid
}

\author{
Mathilde Maillard, J. Boujlel, Philippe Coussot
}

\section{To cite this version:}

Mathilde Maillard, J. Boujlel, Philippe Coussot. Flow characteristics around a plate withdrawn from a bath of yield stress fluid. Journal of Non-Newtonian Fluid Mechanics, 2014, 220, pp.33 - 43. 10.1016/j.jnnfm.2014.08.001 . hal-01117234

\section{HAL Id: hal-01117234 \\ https://hal-enpc.archives-ouvertes.fr/hal-01117234}

Submitted on 16 Feb 2015

HAL is a multi-disciplinary open access archive for the deposit and dissemination of scientific research documents, whether they are published or not. The documents may come from teaching and research institutions in France or abroad, or from public or private research centers.
L'archive ouverte pluridisciplinaire HAL, est destinée au dépôt et à la diffusion de documents scientifiques de niveau recherche, publiés ou non, émanant des établissements d'enseignement et de recherche français ou étrangers, des laboratoires publics ou privés. 


\title{
Flow characteristics around a plate withdrawn from a bath of yield stress fluid
}

\author{
M. Maillard*, J. Boujlel, P. Coussot \\ Université Paris-Est, Laboratoire Navier (ENPC-IFSTTAR-CNRS), Champs sur Marne, France
}

\section{A R T I C L E I N F O}

Article history:

Received 9 May 2014

Received in revised form 21 July 2014

Accepted 1 August 2014

Available online $\mathrm{xxxx}$

\section{Keywords:}

Yield stress fluid

PIV

Dip-coating

\begin{abstract}
A B S T R A C T
Dip-coating is a process widely used in industry to coat a fluid on a solid substrate. The general characteristics of dip-coating for simple liquids (Newtonian) are well known but a lot of applications involve complex fluids. Here we focus on the case of a solid plate coated with a yield stress fluid through the immersion followed by the withdrawal of a plate from a bath of such fluid. We carried out a precise analysis of the force applied to the plate during its withdrawal from the bath, and determined the velocity field developed around the plate in the fluid bath with the PIV technique. It appears that, inside the bath, after a transient regime during the plate withdrawal, a linear force regime is set up, which is associated with a stationary uniform flow (liquid region) settled along the plate while the rest of the material is in its solid regime. We show that the thickness of this liquid region increases slowly with the plate velocity and its value is almost the same for immersion and withdrawal, and independent of a possible period of rest preceding plate motion. Finally we show that the thickness of the liquid region is related to the force in the linear regime.
\end{abstract}

(C) 2014 Published by Elsevier B.V.

\section{Introduction}

Yield stress fluids, such as emulsions, colloids, or foams, form a class of complex materials that flow when submitted to a sufficiently large stress and stay in a solid state otherwise. A lot of operations in our everyday life need to extract an object from a bath of yield stress fluid such as mud, chocolate, paint, cement paste, cream, which leads to coat these objects with a layer of fluid, before spreading or pouring them somewhere else. Various industrial processes (food industry, automobile) rely on this technique for coating or treating the surfaces. The stakes of research in that field are to understand the fluid flow under the effect of the solid displacement to optimize the process parameters: geometrical dimensions of the solid coated and container, distance between the solids, velocity of the object to coat, rheological characteristics of the fluid.

Dip coating with Newtonian liquids (of viscosity $\mu$ ) has been the object of much research and there now exists a solid background of knowledge. To sum up very briefly, most studies in that field focused on the film formation and thickness [1-3]. The value of the film thickness results from a balance between gravity, capillary and viscous effects. In particular, at constant velocity a uniform

\footnotetext{
* Corresponding author.

E-mail address: mathilde.maillard@ifsttar.fr (M. Maillard).
}

film forms along the plate, the thickness $(h)$ of which mainly varies as a power-law of the capillary number $(C a=\mu V / \sigma$, in which $\sigma$ is the fluid surface tension) as long as gravity effects are negligible. The thickness was observed to increase as a power law of $V$ with an exponent $2 / 3$ in the viscous-capillary regime and an exponent $1 / 2$ in the viscous-gravity regime $[1,2]$. However a fundamental aspect of this process with simple liquids is that as soon as the plate motion stops, the fluid drains and the thickness decreases. Much less is known concerning the flow inside the bath. At least it was shown that different flow regimes can be observed, but the liquid flows at any point inside the bath [4], so that the transition from the bath to the surface is smooth.

A very limited number of (phenomenological) studies concerned non-linear fluids. It was shown that elastic effects tend to increase $h[5,6]$. For a shear-thinning behavior a thickness increase [7] or decrease [8] was observed when the power-law index decreases. For yield stress fluids some numerical simulations $[9,10]$ suggested that $h$ increases with $\tau_{c}$. A theoretical analysis [11] in the case of dominant yielding effect (i.e. plastic flow) and negligible gravity effects finally predicted $h \propto \tau_{c}^{2}$. Recently the first experimental study with yield stress fluids showed that the coated layer remains stuck on the plate after its withdrawal, and that the coated thickness is approximately proportional to the yield stress of the material [12]. The thickness at vanishing velocity was found to be equal to $0.3 \tau_{c} / \rho g$. Moreover it was suggested that the coated 
thickness is related to the flow characteristics inside the bath [12]. The present work looks in more details at the characteristics of dipcoating with yield stress fluids. In particular we study in depth the different flow regimes during withdrawal, we analyse in detail the force on the plate as a function of velocity in these different regimes, and we study how this information may be used to deduce the coated thickness, and we describe the flow characteristics inside the bath during the plate withdrawal as this can provide key information for understanding the origin of the formation of the coated layer. Finally we compare these results with those obtained in the case of plate immersion.

The withdrawal of a solid object from a bath of yield stress fluid is a problem related to the displacement of an object through a yield stress fluid. This problem has so far been mainly studied in the case of a compact object (see for example [13]). The displacement of a sphere or a cylinder moving through a yield stress fluid has been studied in depth via theory and simulations assuming an undeformed rigid region beyond some distance from the object [14-19]. Force versus velocity expressions have been deduced and partly confirmed experimentally (although some unexplained discrepancies remain) [20-23]. Basically the force follows a Herschel-Bulkley type expression with an apparent shear rate equal to the ratio of the velocity to an "apparent" sheared thickness proportional to the sphere radius. The drag force on a cylinder [24] and on a disk [25] were recently determined from systematic experiments with well-controlled yield stress fluids.

Only a few works aimed at measuring directly the flow field around the object. Atapattu et al. [20] were the first to measure the tangential velocity profiles around a sphere. Gueslin et al [26] provided an in-depth study of the velocity field but this concerned a thixotropic material for which there can be a further, and maybe dramatic, impact of the intense shear rate close to the object surface. Finally some works [27,28] (for spheres) and [24] (for cylinders) provided new detailed data concerning the velocity field around an object moving through a yield stress fluid. They found a significant discrepancy of the velocity field with that computed from simulations. In particular they remarked an asymmetry on the flow field, the extent of the sheared zone ahead of the object being larger than expected from theory. A significant flow asymmetry was also observed in 2D foam flow around an obstacle by Dollet and Graner [29] which was attributed to elastic effects.

It is likely that an issue in these works, which nevertheless might not explain the observed asymmetry, is the following: although they are generally considered as the flow characteristics of the fluid in the liquid regime, the measurements concern the apparent velocity field which could include both flow in the liquid regime and deformations in the solid regime. This means that new deformations of the material in the solid regime continuously occur around the object and might play a significant role in the process. This conclusion was confirmed by the analysis of the flow field around an object of simpler form, i.e. a plate, penetrating through a bath of yield stress fluid [30]: although the fluid seems to exhibit a complex velocity distribution from the instantaneous velocity field, it was shown that only a layer of small thickness along the plate effectively flows in the liquid regime while the rest of material undergoes small total deformations in the solid regime.

Here we study the withdrawal of a thin plate from a bath of yield stress fluid by analyzing the evolution of the force applied on the plate during its displacement and measuring the velocity field by PIV. First, we present the materials, equipments and procedures used (Section 2). Then, we analyze the different regimes that occur during the withdrawal of the plate looking first at the time evolution of the force applied on the plate (Section 3), then at the velocity field inside the bath (Section 5 ). We also discuss the impact of different parameters (geometry, fluid properties, velocity) (Section 4). Finally, we compare these observations with those corresponding to plate immersion [30,31] (Section 6) before studying the impact of a time of rest between the plate immersion and withdrawal (Section 7).

\section{Materials and methods}

\subsection{Materials}

As yield stress fluids, we used solutions of Carbopol 980 in water at different concentrations (see details on these materials in [32]). Rheometrical tests were carried out with a Bohlin C-VOR rheometer equipped with two circular rough parallel plates to prevent slippage (diameter $4 \mathrm{~cm}$; waterproof sandpaper of average particle diameter $120 \mu \mathrm{m}$ ). Sweep tests were carried out: the stress was increased logarithmically in time (for $3 \mathrm{~min}$ ) and then decreased for the same time. The data in the decreasing ramp are considered as describing the material behavior in steady state. Various data of that type have been published recently (see for example [33]). They showed that these materials behave as simple yield stress fluids without thixotropy. The flow curve representing the steady state shear stress as a function of the shear rate can be very well fitted over four decades in shear rate $\left[10^{-2} ; 10^{2} \mathrm{~s}^{-1}\right]$ by a Herschel-Bulkley model. The resulting behavior in simple shear is $\tau<\tau_{c} \Rightarrow \dot{\gamma}=0$ (solid regime) and $\tau>\tau_{c} \Rightarrow \tau=\tau_{c}+k \dot{\gamma}^{n}$ (liquid regime), where $\dot{\gamma}$ is the shear rate and $\tau_{c}, k$ and $n$ are parameters depending on the material characteristics. For such materials it was shown from MRI measurements that, in contrast with more complex (thixotropic) yield stress fluids, this macroscopic constitutive equation effectively corresponds to their local rheological behavior inside the bulk [32]. For our experiments, we used 9 different Carbopol gels (see [31] for details on material preparation) with $\tau_{c}$ in the range [9-82], the flow curves of the 4 gels mainly used in this study are shown in Fig. 1.

\subsection{Dip-coating}

We studied the dip-coating of a solid plate with the same experimental set-up used for the immersion of a plate in a yield stress fluid [30]. Here a vertical plate initially partially immersed is withdrawn at constant velocity $V$ from a container filled with Carbopol gel. The solid is directly linked to a dual-column testing system (Instron 3365) which controls its position with a resolution of $0.1 \mu \mathrm{m}$. The apparatus is equipped with a $10 \mathrm{~N}$ force sensor able

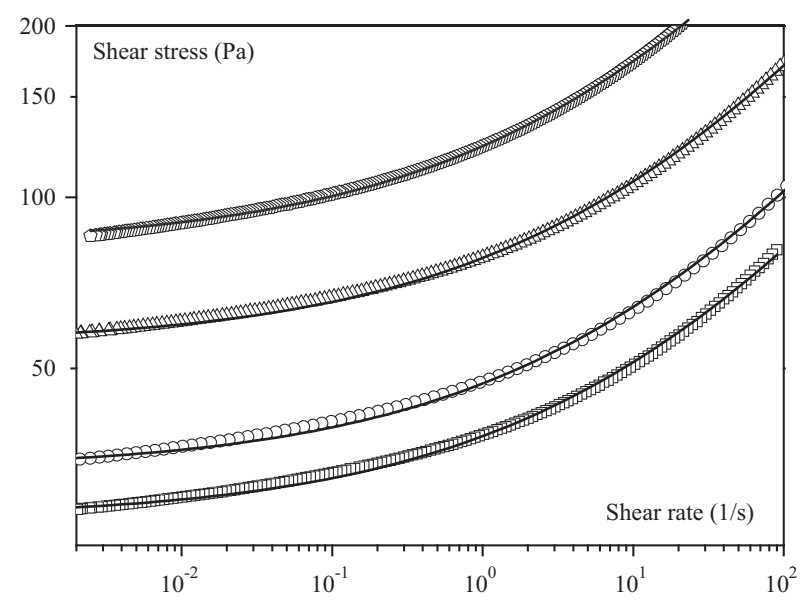

Fig. 1. Flow curves of our different Carbopol gels. The continuous lines correspond to a Herschel-Bulkley model fitted to data with the following parameters $(n=0.35)$ : $\tau_{c}=27 \mathrm{~Pa}, k=10.7 \mathrm{~Pa} \mathrm{~s}^{\mathrm{n}}$ (open squares); $\tau_{c}=33 \mathrm{~Pa}, k=13.8 \mathrm{~Pa} \mathrm{~s}^{\mathrm{n}}$ (circles); $\tau_{c}=55$ $\mathrm{Pa}, k=23 \mathrm{~Pa} \mathrm{~s}^{\mathrm{n}}$ (open triangles) and $\tau_{c}=82 \mathrm{~Pa}, k=40.7 \mathrm{~Pa} \mathrm{~s}^{\mathrm{n}}$ (open pentagones). 
to measure the force applied on the plate with a precision of $\pm 10^{-5}$ of the maximum value. The force applied on the plate during its withdrawal is recorded. The velocity is varied between 0.1 and $17 \mathrm{~mm} \mathrm{~s}^{-1}$. Note that long duration experiments at low velocities with such fluids are often affected by artefacts such as some kind of flow localization along the walls [32] and drying. For this reason we did not attempt to make tests at velocities smaller than $0.1 \mathrm{~mm} / \mathrm{s}$.

Except when mentioned otherwise, the thickness of the plate is $e=1.9 \mathrm{~mm}$, its width $l=7.1 \mathrm{~cm}$ and its length $H=25 \mathrm{~cm}$ (a maximum length of $24 \mathrm{~cm}$ could be coated). In order to avoid wall slip effects we covered the solid surfaces with waterproof sandpaper of average particle diameter $120 \mu \mathrm{m}$, giving an effective roughness of a few tenths of microns, a thickness a priori larger than the elements suspended in the liquid. Three kinds of container were used, two were beakers of diameter $12 \mathrm{~cm}$ and $18 \mathrm{~cm}$ and one was a $10 \mathrm{~cm}$-width and $15 \mathrm{~cm}$-length parallelepiped.

In these configurations, we checked that the flow is independent of the geometrical parameters of the set up as soon as some ratios of the different dimensions are large enough. This point was addressed in [30] in the case of immersion and we discussed it in details in Section 4.1 in the case of withdrawal. Besides, the thickness of the coated layer is always much smaller than the width of the plate (at least 15 times) which supports the assumption of a 2D flow in the plane of observation by PIV (if we assume that the flow inside the bath involves a thickness of the same order, a result confirmed below).

Before each experiment, the plate is first wetted with the Carbopol solution and the excess of material above the roughness is removed. Then the free surface is smoothed. A test is composed of two phases: the plate is immersed at constant velocity in the bath of material then it is withdrawn at the same constant velocity. Except when mentioned the tests were carried out with no time of rest between the plate immersion and withdrawal. Between two successive measures the material in the container is left at rest for some time in order to ensure residual stress relaxation and the material is slightly mixed to prevent the plate from being dipped in the same region of fluid. All measurements are performed at room temperature.

\subsection{Particle Image Velocimetry}

Along with macroscopic force recordings and taking advantage of the transparency of the material, we carried out local measurements of the velocity profiles inside the bath during the withdrawal of the plate with the Particle Image Velocimetry technique (PIV). We used the same technique as in [30]. Polystyrene beads (diameter $80 \mu \mathrm{m}$ ) were dispersed in the bath to be used as markers at a mass fraction of around $0.2 \%$. Their density was very close to that of the fluid to prevent their sedimentation. We checked that their impact on the rheological behavior of the Carbopol solutions was negligible. The motion of the particles was observed in a vertical plane perpendicular to the solid plate and along its central axis, enlightened with a $20 \mathrm{~mW}$ continuous red laser sheet. A CCD camera attached to the container recorded the particles displacements along the plate during all the duration of its withdrawal until the plate disappeared off the picture. Depending of the camera adjustments, the picture size is around $5 \mathrm{~cm}$ height and $6.5 \mathrm{~cm}$ width, namely $552 \times 767$ pixels. 12 pixels stand approximately for $10 \mathrm{~mm}$. Note that when the plate is in the camera picture, there is a large part of the fluid that is not enlightened due to the set up. So the velocity field is determined over a single side of the plate. An experiment represents the study of the plate displacement over a distance of about $15 \mathrm{~cm}$. The movies obtained have a frequency up to 15 frames per second.
The data were analyzed with the commercial software DaVis, with the same process used in our previous work concerning the immersion of the plate in a bath of Carbopol gel (see [30] for more technical details). Each image was divided into squares of correlation of $24 \times 24$ or $36 \times 36$ pixels. On a given stack of images, we used a cross-correlation technique comparing successive images through the determination of the average particles displacements between two pictures in every correlation square. The first image was compared to the second one, the second one to the third one and so on, taking into account all the images of a given stack. Then all the correlations were averaged to give the final velocity field of the stack of images. In these fields, there is one velocity per correlation square.

The flow fields were obtained by averaging the pictures taken during a $1 \mathrm{~cm}$ displacement of the plate. Thus, the number of images per stack used to compute an averaged velocity field varies for each test and decreases with the plate velocity. This displacement of $1 \mathrm{~cm}$ was chosen so as to have enough information on the flow and a sufficient particle displacement in each correlation square while limiting the noise around the data for the largest velocities. Using a constant displacement to compute the velocity field in the fluid whatever the plate velocity allows easier comparison between the data.

\section{Flow regimes}

\subsection{Coated layer uniformity}

First, the plate is partially immersed in the bath and then withdrawn at constant velocity. A first striking observation is that whatever the plate velocity and the gel yield stress, a coated layer forms on the plate when the withdrawal starts. The coating ends when the plate is no more linked to the bath of fluid, which happens when the plate is around $3 \mathrm{~mm}$ above the free surface in our range of material yield stresses. Then, contrary to what happens with simple liquids, no drainage is observed once the plate is out of the bath. The mass of fluid coated on the plate can be easily recorded thanks to the force machine since once the plate is out of the container the only force applied onto the plate is the weight $W$ of the fluid stuck on the plate.

We repeated the full process for different initial immersion depths $L_{0}$. Fig. 2 presents the variation of $W$ as a function of $L_{0}$. When $L_{0}$ is larger than about $4 \mathrm{~cm} \mathrm{~W}$ appears to be proportional to $L_{0}$. Since $W$ is proportional to the volume of coated fluid, this means that the coated layer has a uniform thickness $h$. For smaller

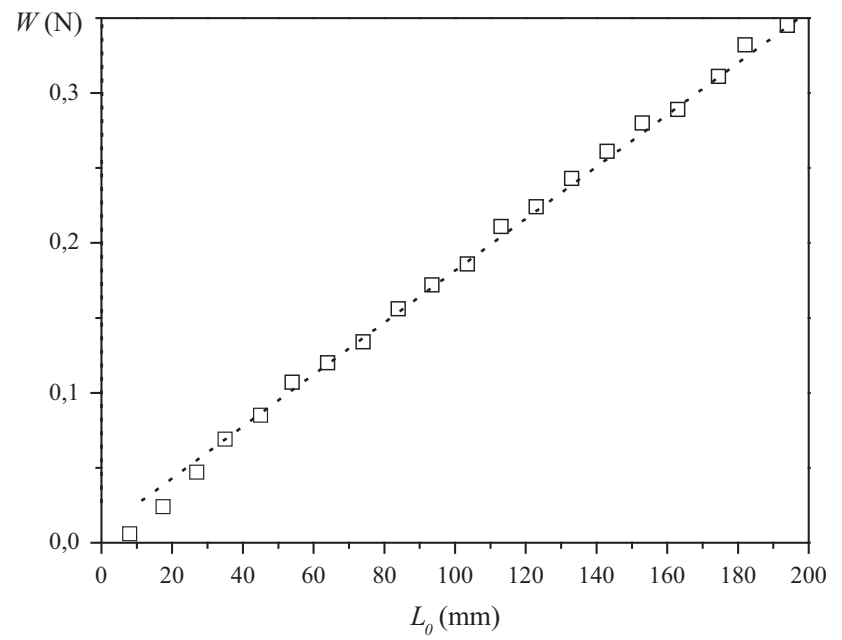

Fig. 2. Weight $(W)$ of fluid $\left(\tau_{c}=33 \mathrm{~Pa}\right)$ coated on the plate after its withdrawal versus the initial immersed depth $\left(L_{0}\right)$ for $V=10 \mathrm{~mm} \mathrm{~s}^{-1}$. 
$L_{0}$, edge effects play a significant role, i.e. the coated layer thickness increases progressively from 0 to $h$. A similar effect occurs at both tips although not with the same shape, as described in the following section.

Under these conditions it is possible to get rid of the contribution of the fluid coated on the edges and thus determine the thickness $h$. Considering that a layer of uniform thickness $h$ is coated all around the plate, we have $h=(1 / \rho g P)\left(d W / d L_{0}\right)$, in which $\left(d W / d L_{0}\right)$ is taken in the linear part of the curve, $P=2(e+l)$ is the perimeter of the plate, and $\rho$ the fluid density. All the coated thicknesses presented in the following are determined with this method, using five mass measurements in the range $9<L_{0}<21 \mathrm{~cm}$. The uncertainty on such data is of the order of $15 \%$.

\subsection{Force evolution}

In order to get more information on the formation of the coated layer in time, we followed the force $F$ applied on the plate during all its withdrawal from the bath of yield stress fluid, as a function of the immersed depth of the plate $L(L<H)$. More precisely, $L$ is the distance between the fluid free surface and the lower tip of the plate. Before immersing the plate, the force $W$ is reset to zero. With this procedure the recorded force $F$ does not take into account the constant contribution of the plate weight and strictly represents the result of the interaction between the fluid and the plate.

We varied the yield stress of the Carbopol gel, the plate velocity and the depth of the initial immersion of the plate, but the results were qualitatively the same. A typical result for $F$ is represented in Fig. 3(a). The plate is partially immersed at a depth larger than $8 \mathrm{~cm}$ in order to exceed the uniform plate coating threshold (see Fig. 2). The different stages of the force curve during immersion have been discussed by Boujlel et al. in [30]: after a small initial step when the plate first reaches the fluid, $F$ increases linearly with $L$. Here we focus on the other (five) regimes identified in the force curve during the plate withdrawal (see Fig. 3(a)).

After the immersion stage, the plate is immediately withdrawn. Since during the immersion the fluid applies on the plate a force directed upward, $F$ is positive at the first instant of withdrawal (regime (i)). When we start to withdraw the plate, a transient regime occurs during which the force applied by the bath on the plate quickly decreases and becomes negative (regime (ii)). When the plate has been withdrawn of a few centimeters, there is a linear phase where $F$ is proportional to $L$ (regime (iii)). When the tip of the plate reaches the free surface, another transient phase appears (regime (iv)) where $|F|$ decreases faster so as to finally reach a plateau associated to complete exit (regime (v)).

\subsection{Analysis}

Looking at the form of the coated layer on the plate (Fig. 3(b)), we can link its different parts to the evolution of $F$ (Fig. 3(a)).

1. In regime (ii), the force decrease corresponds to the inversion of the deformations previously induced by the immersion flow around the plate. At the same time the plate coating begins, inducing a negative force. $F$ finally becomes negative when the upward flow is settled and then starts to increase when its variation due to plate coating is dominant.

Note that the final coated length is smaller than the total plate displacement in the bath by about $8 \mathrm{~mm}$. It is caused by the existence during immersion of an inversed meniscus of the free surface around the plate. This induces a slight step between the

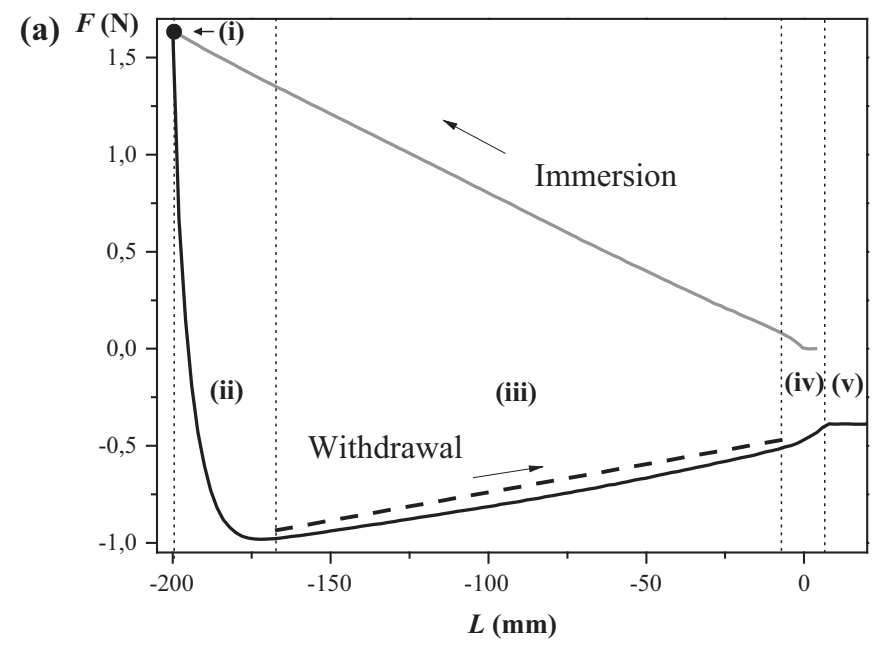

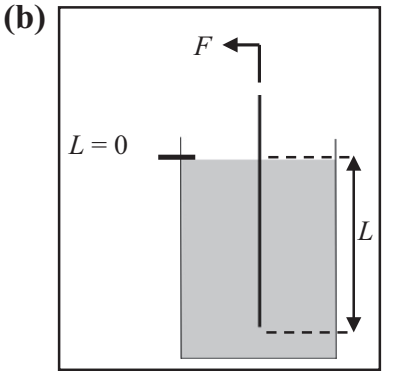

(i)

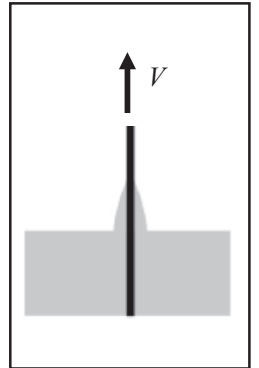

(ii)

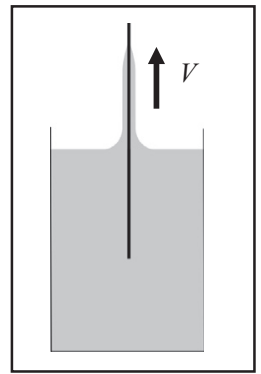

(iii)

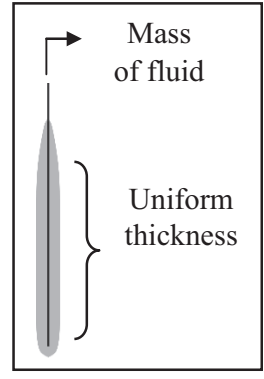

(v)

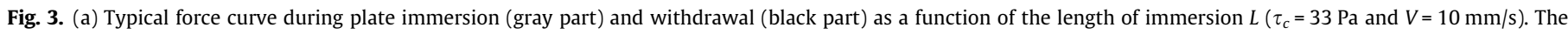

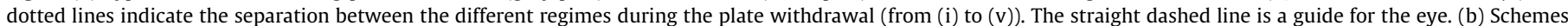

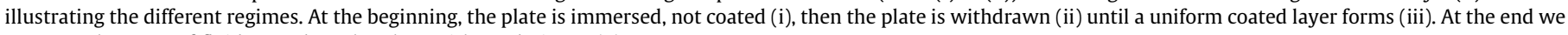
measure the mass of fluid coated on the plate without drainage (v). 
free surface and the contact line so that the effective coating begins as soon as this contact line reaches the free surface. Then an upward meniscus appears between the plate and the free surface (see Fig. 3(b)(ii)) and a fluid layer forms on the part of the plate that is emerging from the bath.

For large yield stresses, we observe a sort of hollow in the free surface on both sides of the upward meniscus around the plate. This effect is not represented on the schemes as it happens in specific situations. In these cases, the upward meniscus due to the withdrawal of the plate do not overcome the transverse extension of the inward meniscus, resulting in the existence of hollows.

2. Then, during regime (iii), the force curve presents a linear phase which corresponds to the coating on the plate of a layer of uniform thickness $h$ (see scheme (b)(iii)). In this regime, the forces applied on the plate are due to:

- capillary effects, $f_{c}$, at the fluid-plate-air contact line,

- buoyancy force on the immersed part of the plate, $f_{b}=-\rho g$ Lle,

- viscous force due to fluid flow around the plate. It can be expressed as the sum of a term due to edge effects $f_{e}$ and a term due to the uniform flow along the plate $\left(f_{v}=2 \tau L l\right)$, where $\tau$ is the shear stress along the plate,

- mass increase of the coated fluid, $f_{w}=2 \rho \operatorname{glh}\left(L_{0}-L\right)$; note that here we suppose for the simplicity of the expression that the coating is uniform, but this does not change the final result of our calculation.

A scheme of the dip-coating process is represented in Fig. 4. We distinguish two parts in the fluid whether it is above or below the free surface, simplifying the zone around the meniscus.

Quickly after the beginning of the plate withdrawal from the bath, i.e. during regime (iii), we observe that the shape of the air-fluid interface around the contact line remains fixed. Thus the corresponding force, i.e. associated with capillary effects, is constant. On the other hand, if we assume that the layer of material coated on the plate out of the bath constitutes a rigid body attached to the plate, the buoyancy force is simply proportional to the volume of the immersed part of the plate. As the plate section is constant, this force is proportional to $L$. Since the mass of coated fluid is proportional to $L$ in regime (iii), the corresponding weight varies in the same way.

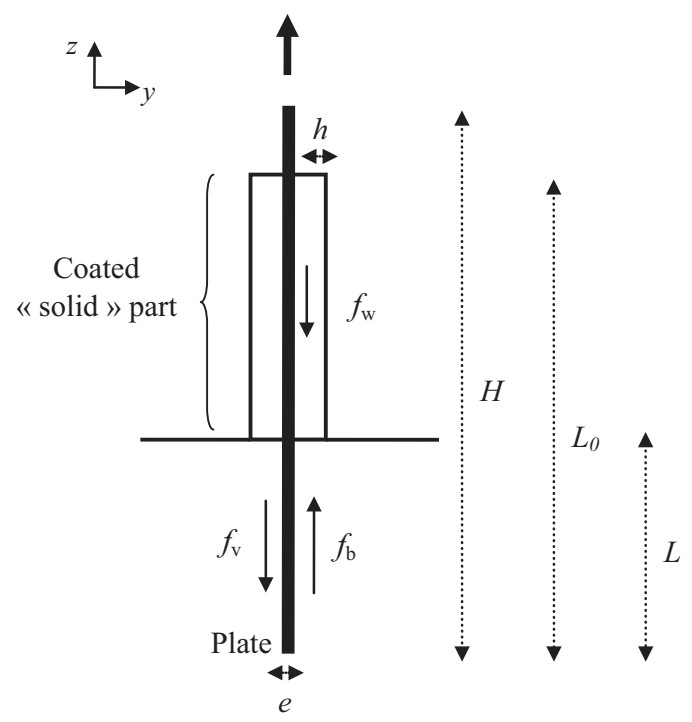

Fig. 4. Schematic representation of the fluid distribution around the plate during its withdrawal from the bath, and of the non-constant forces acting during the process. The continuous line represents the schematic shape of the free surface.
Since the total force, which is the sum of the four above forces, is proportional to $L$ in regime (iii) while one of them is constant (and thus negligible in this regime) and two of them are also proportional to $L$, we deduce that the last one, i.e. the viscous force, is either negligible, which is unrealistic, or proportional to $L$ too. This suggests that, as for the immersion flow (see [30,31]), edge effects are constant and the flow along most of the immersed part of the plate is uniform and constant during plate withdrawal.

In the stationary regime the derivative of the total force with regards to the immersed length writes:

$$
\frac{d F}{d L}=2 \tau l-\rho g e l-2 \rho g l h
$$

This equation shows that in the linear regime, if the coated thickness is known, the viscous stress along the plate in the uniform region can be deduced from the measure of the slope of the force versus immersed depth curve. This point is discussed in details in Section 6.

3. Finally, around $1 \mathrm{~cm}$ before the tip of the plate reaches the free surface, the regime (iv) starts: the force increases more rapidly because the viscous force disappears when the plate leaves the bath. It is the end of the linear phase, the coated layer thickness decreases from $h$ to 0 .

4. At the end, in regime (v), the coated plate is completely out of the bath and contrary to Newtonian or viscous fluids, there is no drainage, the fluid stays stuck on the plate (see scheme (b)(v)) and the force recorded, which is now constant, only corresponds to the weight of the fluid coated on the plate.

Note that the fourth phase which represents the transition between the linear and the constant regimes does not strictly end when $L=0$ due to the presence of a small amount of fluid linking the plate and the free surface for some time before this connection breaks itself.

A detailed study of the force curves show that the extensions of the regimes (ii) and (iv), i.e. the lengths over which they take place, significantly increase with the yield stress - they are multiplied by $30 \%$ when $\tau_{c}$ is multiplied by 3 - and slightly increase with the plate velocity - they increase by $60 \%$ when $V$ is multiplied by 100 - while they are independent of the immersion depth $L_{0}$ provided it is larger than the sum of these extensions. In our ranges of study for both $V$ and $\tau_{c}$, the extension of regime (ii) varies between 22 and $50 \mathrm{~mm}$ and that of regime (iv) varies between 6 and $20 \mathrm{~mm}$, so the total transient extension varies between 28 and $70 \mathrm{~mm}$. This means that the extension of the uniform coated layer also depends of $\tau_{c}$ and $V$, in the opposite way of the uniform coated thickness (see below). Globally speaking this implies that the uniform regime (iii) is less quickly settled when the coated thickness to reach is larger.

\section{Impact of boundary conditions and material characteristics on the coated layer thickness}

\subsection{Impact of geometrical conditions}

Our experiments were carried out with one unique plate and three different sizes of containers after having checked out the impact their dimensions had on the flow induced by the motion of the plate. In that aim we can rely on the observation that, as it appears from the force measurements, the flow induced by the plate during its withdrawal is roughly the opposite of the one induced by immersion (see Fig. 3), so its characteristics are expected to be similar to the one observed during immersion and presented in [31]. In particular, extrapolating the conclusions in immersion we can assume that the flow in withdrawal is as well 
independent of the container and plate dimensions under some non-binding size ratio conditions determined in [31]. The most important condition is that the distance between the plate and the container size has to be larger than $2 \mathrm{~cm}$. It was also found that the flow is independent of the geometrical characteristics if the ratios $e / l$ and $e / H$ are smaller than 0.032 , which is the case in the present tests. These results have been checked for a container diameter up to $240 \mathrm{~mm}$.

To study specifically its impact on the coated layer, we carried out tests by varying the plate thickness between 1.5 and $4.3 \mathrm{~mm}$. We confirmed that whatever the plate thickness, $F$ varies in the same way as described above with the same five regimes and we observed that the coated layer thickness in the uniform region appears to vary negligibly with regards to the uncertainty on each independent measurement. As a consequence in the following we present only data obtained with one plate thickness. The condition $H>4 \mathrm{~cm}$ discussed previously is also added to ensure a uniform coating.

\subsection{Impact of the plate velocity}

As seen previously there is no drainage once the plate is out of the bath, it is thus possible to compute the uniform film thickness $h$. Fig. 5 presents the evolution of $h$ for three gels with yield stress in the range [27; $82 \mathrm{~Pa}$ ] and plate velocity between 0.2 and $17 \mathrm{~mm} \mathrm{~s}^{-1}$.

We can remark that there is a significant scatter on data especially at high yield stress and small velocities likely because in those cases the coating is more easily affected by the imperfections of the experiments such as a slight deviation of the plate from the vertical position or an imperfect horizontal free surface. The final recorded weight for the same test appeared to fluctuate within $10 \%$ around its mean value. This might be due to slight variations in the plate inclination and slight heterogeneities of the fluid state in the bath. Additional uncertainties in the coated height and the mathematical process for estimating of the fluid thickness are around $5 \%$. Finally the uncertainty on the whole technique for determining the fluid thickness is about $15 \%$. It was suggested in [12] that looking at the average tendency of the curves, all the $h$ versus $V$ curves have a shape similar to that of a flow curve following a Hershel-Bulkley behavior: a stress plateau at small velocities and a stress increase at large velocities. The existence of a thickness plateau of the order of one millimeter at low velocities implies that for a given fluid a coated layer forms whatever the plate velocity, with a thickness larger than a critical thickness $h_{c}$.

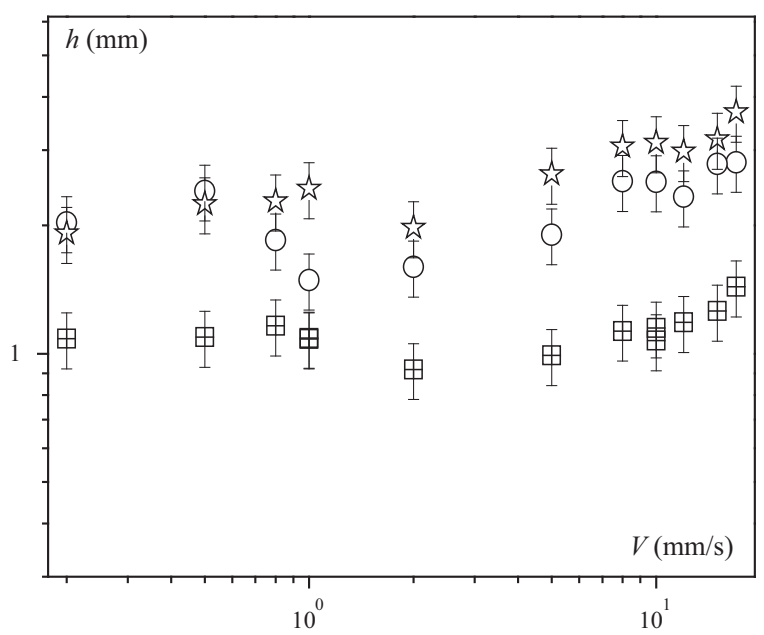

Fig. 5. Coated layer thickness $h$ after dip-coating process as a function of the plate velocity for different yield stress fluids: (crossed squares) $27 \mathrm{~Pa}$, (circles) 55, (stars)

\subsection{Impact of the fluid yield stress}

Fig. 5 shows that $h_{c}$ increases with the fluid yield stress. This effect may be studied directly through the evolution of $h$ as a function of $\tau_{c}$ and $V$ (see Fig. 6). We note that for each velocity level $h$ is roughly proportional to $\tau_{c}$. Moreover, $h$ is much more sensitive to an increase in $\tau_{c}$ than to an increase in $V: h$ is almost multiplied by 3 when $\tau_{c}$ is hardly multiplied by 9 , whereas $h$ is barely doubled when $V$ increases over 2 decades.

\section{Velocity field}

The force measurements gave us information on the way the uniform coating thickness $h$ evolves with the experimental parameters and suggested some characteristics of the flow in the bath. In order to confirm these results and be more precise, we carried out direct local measurements inside the fluid by PIV.

For each velocity, the plate withdrawal was filmed and the movies were analyzed to determine the time evolution of the velocity field along the plate in the bath. Here the container made of plexiglas is $15 \mathrm{~cm}$ long, $10 \mathrm{~cm}$ wide and $30 \mathrm{~cm}$ high. Considering the conclusions we drew concerning the non impact of the set-up dimensions, this choice has no influence on the following results.

\subsection{General observations}

Fig. 7 presents the typical aspects of the flow field developed around the plate at three different localizations: (a) near the free surface, (b) along the plate in the bath, (c) around the bottom tip of the plate in the bath. In (b) and (c), the free surface is located around $5 \mathrm{~cm}$ above the flow field and the camera was moved to observe the free surface in (a). Far from the plate tip and from the free surface (Fig. 7(b)), the fluid velocity is apparently uniform along the plate. In this vertical domain close to the plate and of uniform thickness, the fluid is submitted to a large shear rate, whereas $6 \mathrm{~mm}$ nearby, the shear seems negligible. At some distance from the plate the vertical velocity $v$ is negative, which is due to the downward motion of the fluid to replace the volume left free by the plate departure.

The region around the bottom tip (Fig. 7(c)) is deformed in each direction in a quite large area and in particular in the direction transverse to the plate. It is interesting to note that at about $1 \mathrm{~cm}$ under the plate the fluid apparently rotates and finally flows downwards where the plate is moving upwards. This likely results from the fact that once the plate has passed, the fluid has to fill the

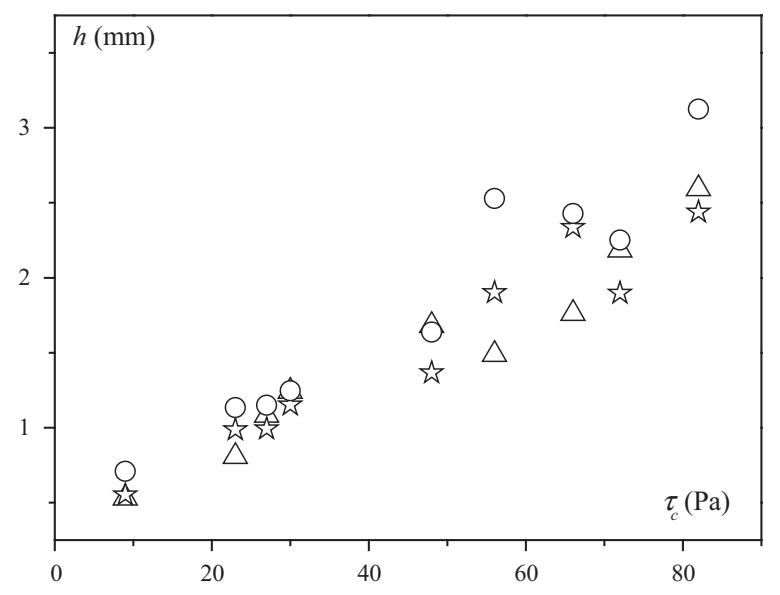

Fig. 6. Coated layer thickness as a function of yield stress for different velocities: (circles) $10 \mathrm{~mm} / \mathrm{s}$, (stars) $5 \mathrm{~mm} / \mathrm{s}$, (triangles) $1 \mathrm{~mm} / \mathrm{s}$. 


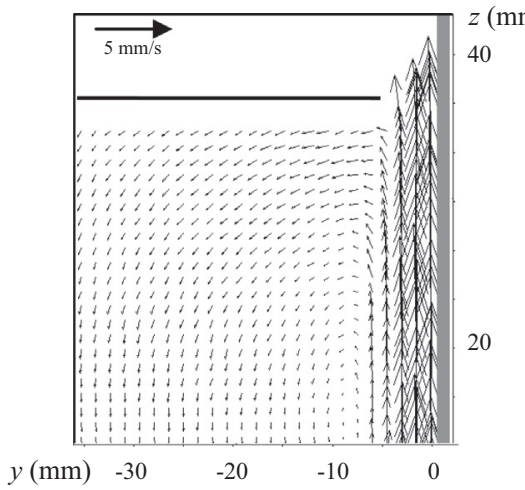

(a)

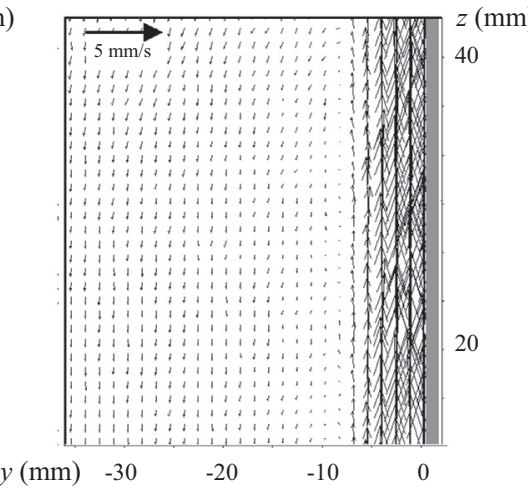

(b)

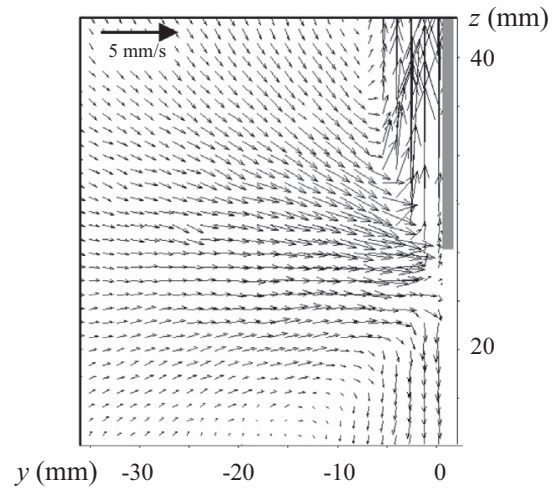

(c)

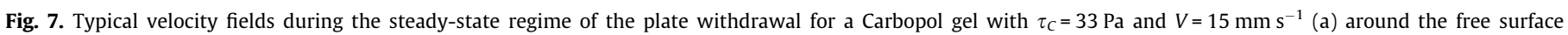
symbolized by the line, (b) along the plate in the bath, and (c) around the bottom tip. The gray area represents the average plate position.

gap left by the plate withdrawal: two rolls of opposite directions form in the bath, with different extensions and values. This region of downward flow, called a negative wake, has been observed behind the motion of objects through non-Newtonian fluids in particular with bubbles [34] and cylinders [18].

Finally, Fig. 7(a) presents the velocity field around the free surface. The liquid layer surrounding the plate divides into two parts once arriving near the free surface: one is taken by the plate and is then coated on it, while the other part of the liquid layer, beyond some distance from the plate, stays in the bath. This velocity field also shows the fluid recirculation at the free surface. No drainage in the coated layer was observed during the experiments so one can assume that the liquid layer comes back to the solid state in the meniscus area even if it is not possible to specify when this occurs. Likewise, the liquid part that stays in the bath becomes solid again before going downwards within the recirculating flow.

In the following we focus on the profiles, along the horizontal direction, of the vertical velocity $v$ (spatially averaged over $1 \mathrm{~cm}$ ).

\subsection{Evolution in time of the velocity profiles (at fixed position along the plate)}

Fig. 8 shows the time evolution of the profiles of the vertical velocity $v$ along the plate at different fixed positions from the plate tip: $10,6,2$ and $-1 \mathrm{~cm}$. In each case, four profiles corresponding to a plate displacement of $4 \mathrm{~cm}$ are drawn. As the camera field does not move during one experiment, the length $\left(L_{0}-L\right)$ of the plate displacement before the first profile - out of the four presented is computed depends of the position under study. The set of profiles shown in Fig. 8 is determined during plate displacements between 7 and $21 \mathrm{~cm}$. These profiles superimpose well, which means that after the transient regime during which the flow direction changes, the flow is stationary all along the plate, in the frame of reference attached to the plate.

We can also look at the evolution of the vertical velocities as a function of the lateral distance from the plate during the plate withdrawal, in a $1 \mathrm{~cm}$ strip fixed in the frame of reference of the container (see Fig. 9), from the beginning of the plate withdrawing. The time evolution can be divided into three parts. First, there is a transitional part over a $5 \mathrm{~cm}$ plate motion during which the velocity gradient near the plate increases and the extension of the sheared domain significantly decreases. Then a steady uniform flow is reached, which lasts over $10 \mathrm{~cm}$ of plate motion. The profile exhibits a velocity plateau far from the plate, and an almost constant shear rate over several millimeters near the plate. Finally, when the plate tip approaches (from about $4 \mathrm{~cm}$ ) the observation strip, the flow deviates from the uniform profile. Once the plate

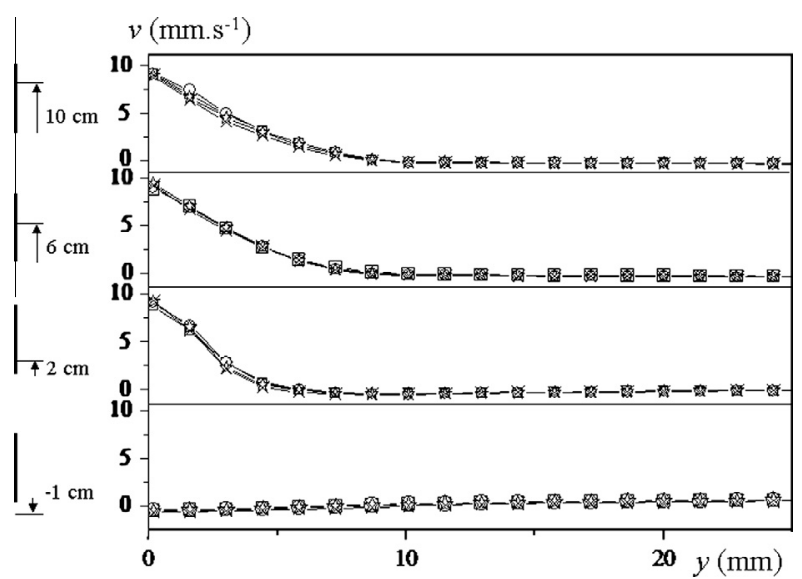

Fig. 8. Velocity profiles (as a function of the distance from plate) transverse to the plate during its withdrawal for $\tau_{c}=33 \mathrm{~Pa}$ at $V=10 \mathrm{~mm} / \mathrm{s}$ at four fixed distances from the plate tip, from top to bottom: $10 \mathrm{~cm}, 6 \mathrm{~cm}, 2 \mathrm{~cm}$ and $-1 \mathrm{~cm}$. For each fixed distance from the tip, four successive velocity profiles are represented, separated one of the other by a $1 \mathrm{~cm}$ displacement of the plate: the first profile is represented with open squares, the second profile in open circles, the third one in open stars and the forth one in crosses. The first profile at $10 \mathrm{~cm}$ from the tip is taken after a plate displacement of $7 \mathrm{~cm}$, the first profile at $6 \mathrm{~cm}$ is taken after a plate displacement of $11 \mathrm{~cm}$, the first profile at $2 \mathrm{~cm}$ is taken after a plate displacement of $15 \mathrm{~cm}$ and the first profile at $-1 \mathrm{~cm}$ is taken after a plate displacement of $18 \mathrm{~cm}$.

tip is away there is a slight downward fluid motion below the plate since the fluid tends to replace the volume left by the coated plate (see Fig. 7(c)), and when the plate tip is at a distance larger than $1 \mathrm{~cm}$ the fluid has turned back to rest.

The timing of the regimes of variation of the velocity profiles correspond rather well to those of the regimes of variation of the force during withdrawal: the transient stage for the velocity profile is associated with regime (ii) for the force, then the stationary velocity profile distribution is associated with the linear regime (iii). This confirms the consistency of our above analysis of the whole process.

\subsection{Stationary velocity profiles}

A typical velocity profile (averaged over the stationary profiles) of the velocity along the plate as a function of the distance from the plate, $(v(y))$, exhibits an almost perfect plateau at some distance, say for $y>15 \mathrm{~mm}$ (see Fig. 9). This plateau has a negative velocity $(\Delta v)$, opposed to that of the plate and much smaller: $-\Delta v \ll V$. In our data range, $\Delta v / V=3.2 \pm 0.7 \%$, which is in the same proportion as during immersion and $\Delta v / V$ increases with the velocity. This 


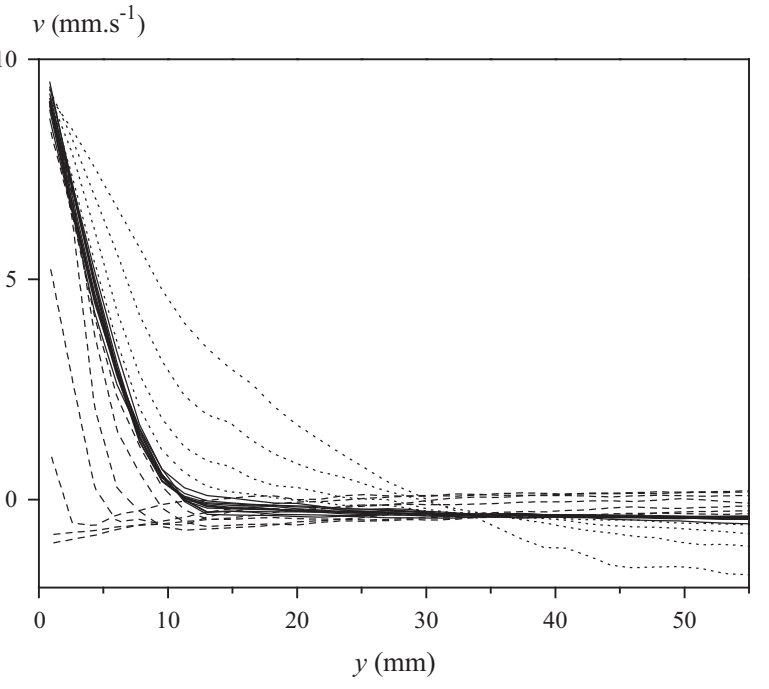

Fig. 9. Time evolution of the vertical velocities as a function of the lateral distance from the plate during the plate withdrawal for $\tau_{c}=33 \mathrm{~Pa}$ and $V=10 \mathrm{~mm} / \mathrm{s}$, computed and averaged in a $1 \mathrm{~cm}$ strip perpendicular to the plate, fixed in the camera field, located at $16 \mathrm{~cm}$ from the plate tip at the beginning of the flow. Each velocity profile corresponds to a $1 \mathrm{~cm}$ displacement of the plate. The 5 first velocity profiles are computed over a $5 \mathrm{~cm}$ plate displacement and are in dotted lines, the 9 following ones are in continuous line, and the 7 final ones are in dashed lines.

slight downward motion of the fluid is needed to fill the volume left vacant when the plate is withdrawn. However the variation of volume cannot be explained by the replacement of the plate only - if so, $\Delta v / V$ would be around $0.1 \%$. The larger value obtained for $\Delta v / V=3.2 \pm 0.7 \%$ mainly results from the fact that the plate induces an upward motion of a fluid layer of thickness of several millimeters, which is consistent with our observations concerning the coated thickness on the withdrawn plate, i.e. extracted from the bath (see Section 4).

The continuous lines in Fig. 9 also show the existence of two regions differently sheared depending on the distance to the plate. The first region, near the plate, is strongly sheared, contrary to the one localized in the plateau region, that is over $15 \mathrm{~mm}$ from the plate, which is not sheared. Thus there is a similarity of the velocity fields along the plate in the immersion and withdrawal cases. If we rely on the detailed analysis of the history of deformation undergone by fluid elements during immersion [30] we can conclude that the region of uniform flow along the plate likely corresponds to the liquid region in the bath of fluid while the rest of the material is essentially deformed in its solid regime. We can also remark that the shear rate near the plate, given by the slope of the profile, is approximately uniform over more than $5 \mathrm{~mm}$.

In order to look at the steady velocity profile in the liquid regime along the plate we compute the average velocity profile estimated over the uniform profiles. Fig. 10 presents the averaged velocity profiles during the plate withdrawal, scaled by the plate velocity for different plate velocities. The liquid and solid zones are represented in broad outline. The thickness of the liquid area is noted $\lambda$. At first sight, the plate velocity has only a slight impact on the shape of the averaged velocity profile, and the scaled velocity profiles are similar, all presenting a plateau above $15 \mathrm{~mm}$ from the plate and a constant shear rate near the plate. In particular, it means that the thickness of the liquid layer does not depend much on the plate velocity.

\subsection{Comparison with the plate immersion}

As expected regarding the force curves, the flow formed around the plate during its withdrawal is very similar to the one formed $v / V$

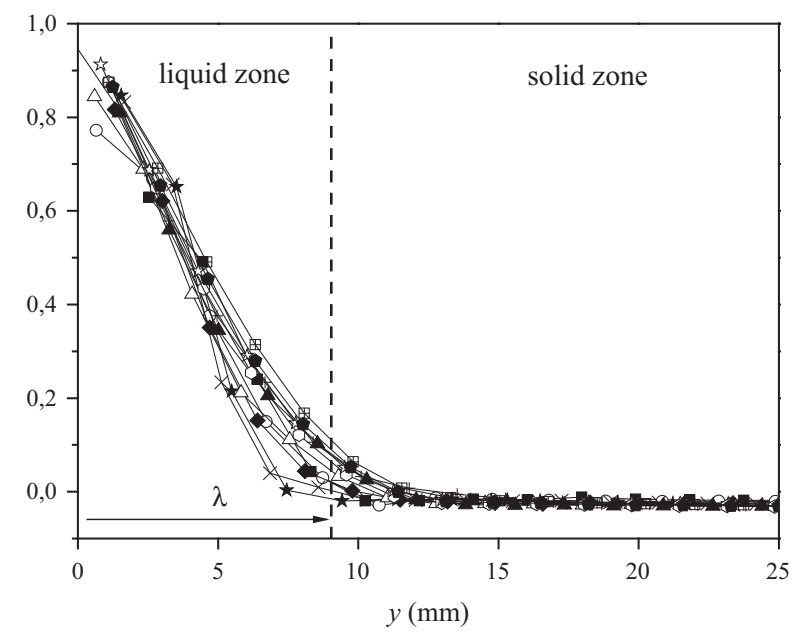

Fig. 10. Average uniform scaled velocity profiles as a function of the distance from the plate for a Carbopol gel $\left(\tau_{C}=33 \mathrm{~Pa}\right)$ and different plate velocities: 0.1 (filled squares), 0.2 (open circles), 0.5 (filled stars), 0.8 (crosses), 1 (filled diamonds), 2 (empty triangles), 5 (filled triangles), 8 (empty hexagons), 10 (empty stars), 12 (filled pentagons), 15 (crossed squares), 17 (vertical crosses). The dashed line schematically separates the liquid and solid zones.

during its immersion notwithstanding the direction, including edge effects and uniform region along the plate [30].

As for immersion, the Carbopol gel flow is stationary in the frame of reference of the plate but it takes more time for the flow to become stationary during the plate withdrawal than during immersion. Such result is confirmed by the force variations in time (see Fig. 3): the transient regime at the beginning of the immersion lasts approximately $10 \mathrm{~mm}$ while the one during the withdrawal lasts twice more time. This results from the fact that during immersion a part of the fluid deformations essentially corresponds to elastic deformations in the solid regime at some distance from the plate. When the withdrawal starts, at the end of the immersion phase, it tends to also induce deformations in the solid regime but in the opposite direction. Before reaching steady-state the plate motion must first inverse previous deformations, which requires a longer distance of displacement.

Then, a liquid layer of uniform thickness forms along the plate during its immersion and its withdrawal. In both cases, their thickness slightly increases with the plate velocity. Fig. 11 presents and compares these variations. At first sight the velocity profiles appears to be made of two parts: one in which the velocity almost does not vary and one region in which the velocity significantly varies along a horizontal axis. It is difficult to determine the exact position of the interface between these two regions. We adopted the following technique: in each of the regions we drew a line over the two last data points clearly situated in this region at the approach of the interface. We thus could estimate $\lambda$ as the point of intersection between these two straight lines. We consider that the uncertainty on this evaluation is around $15 \%$.

Fig. 11 shows that whatever the direction of motion of the plate the same tendencies are observed. We have a kind of plateau at small velocities and a slight increase at larger velocities, as for a Hershel-Bulkley model. The liquid layer thickness in immersion is slightly smaller than during the withdrawal, say by a factor about $12 \%$. This could be explained by the fact that globally, it is easier to withdraw the plate from the bath of fluid than to immerse it since in the first case the plate pushes a large part of the bath of fluid and liquefies a fraction of it, whereas during withdrawal the resistance to the plate motion is weaker as the plate faces the air through the free surface. 


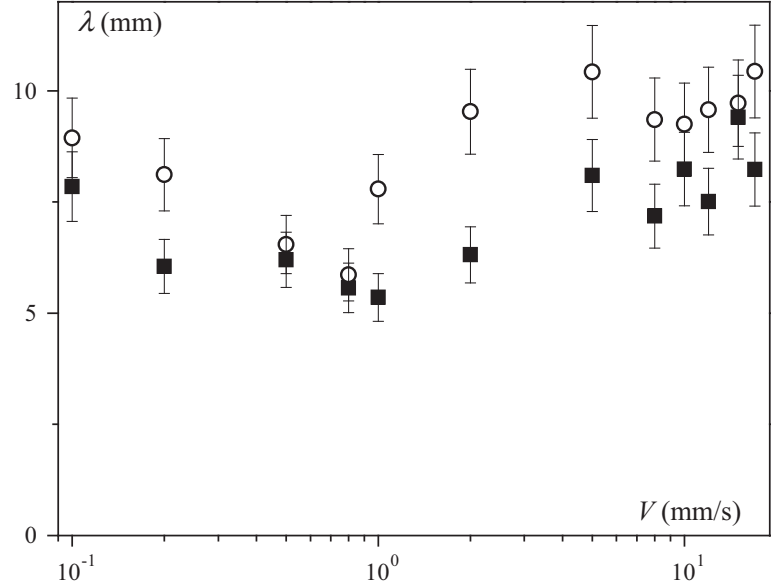

Fig. 11. Liquid layer thicknesses $\lambda$ as a function of the plate velocity $V$ during plate immersion (black squares) and withdrawal (circles) for $\tau_{C}=33 \mathrm{~Pa}$.

It is remarkable that the tendency with the plate velocity is the same for the liquid layer thickness and for the coated layer thickness (see Section 4.2). As already remarked in [12] there finally seems to be an empirical factor, around 0.3 for our data, which links the coated thicknesses to the liquid ones independently of the velocity level. This factor is related to the separation of the fluid volume into two parts (one part which stays in the bath and the other which is coated on the plate) at the approach of the free surface, as may be seen in Fig. 7(a).

\section{Shear stress along the plate}

Due to the uniform flow in the liquid region along the plate, we were able to determine the viscous shear stress at the plate from force measurements (see Section 3.3). Fig. 12 presents the evolution of the shear stresses as a function of the plate velocity, during the plate immersion and withdrawal. In immersion, the shear stresses along the wall were measured with the help of a similar force analysis [31].

The tendencies observed for the shear stresses in the withdrawal and immersion regimes are the same, they slowly increase with the plate velocity and significantly increase with the fluid

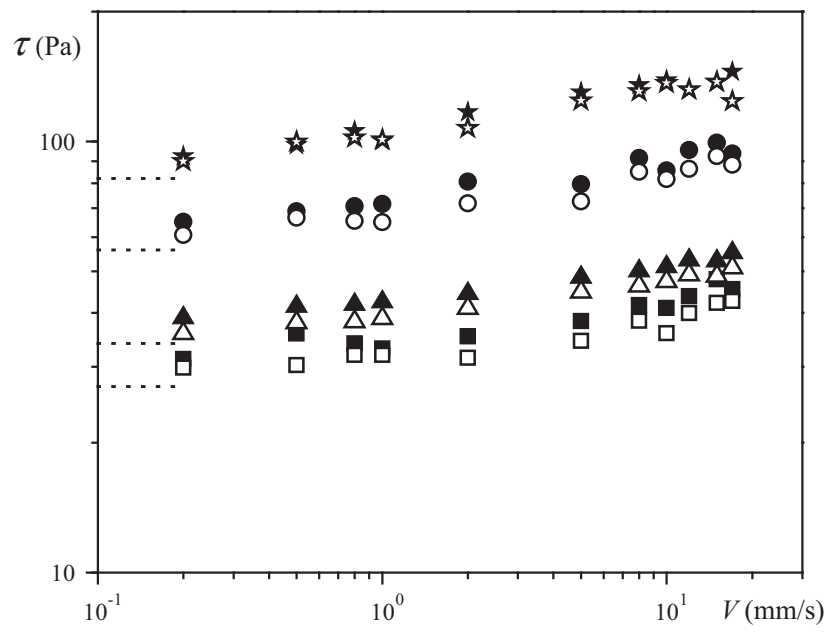

Fig. 12. Viscous shear stress along the plate $\tau$ as a function of the plate velocity $V$ during immersion (full symbols) and withdrawal (open symbols) for $27 \mathrm{~Pa}$ (squares), $33 \mathrm{~Pa}$ (triangles), $56 \mathrm{~Pa}$ (circles) and $82 \mathrm{~Pa}$ (stars). The dashed lines are located at each material yield stress. yield stress. Then, the shear stresses in immersion are slightly larger that the one in withdrawal by around $7-8 \%$. These observations are consistent with the results concerning the liquid thickness: if the shear rate is approximately homogeneous in the liquid region, for the same boundary condition (plate velocity) and using the Herschel-Bulkley behavior, a larger sheared layer leads to a smaller stress.

It is interesting to note that both the immersion and withdrawal shear stresses apparently tend to the fluid yield stress $\tau_{c}$ at vanishing velocity (see Fig. 12). Thus, as for the immersion experiment [31], the computation of the shear stress along the plate may be used to estimate the material yield stress.

For each plate velocity, using the Herschel-Bulkley law, it is also possible to compute the shear stress along the plate with the gradient velocity along the plate deduced from the vertical velocity profiles.

Fig. 13 compares the values obtained with this technique to the previous values computed from the force measurements for $\tau_{c}=33 \mathrm{~Pa}$.

It is remarkable that the data coming from these two methods superimpose very well in the case of the immersion. For the withdrawal, the computation with the velocity gradients again gives data lower than for the immersion, but there are around 5-6\% larger than the one deduced from the force curves.

\section{Impact on the flow of a time of rest}

Since we previously mentioned that the solid deformations play a role in the characteristics of the withdrawal process, at least during the transient stages, it is important to study the influence of a time of rest between immersion and withdrawal, which may have an impact on the initial state of the material just before withdrawing

We looked at the impact of different rest periods (up to $120 \mathrm{~s}$ ) with the same material as before. The force curves were recorded as in Fig. 3(a), the coated layer thicknesses were determined using the method presented in Section 3.1 and the velocity fields were determined with the PIV technique as in Section 5. Note that the PIV technique requires to average the fluid displacement over several images so as to compute a relevant field and to reduce the data noise. This means that this technique has limitations to describe fast transient regimes. In the relaxation case, averaging the velocity field over only 3 images $(1 / 5 \mathrm{~s})$ gives a peak at $0.75 \mathrm{~mm} / \mathrm{s}$ that is $15 \%$ of the plate velocity, instead of $0.43 \mathrm{~mm} / \mathrm{s}$ with 10 images

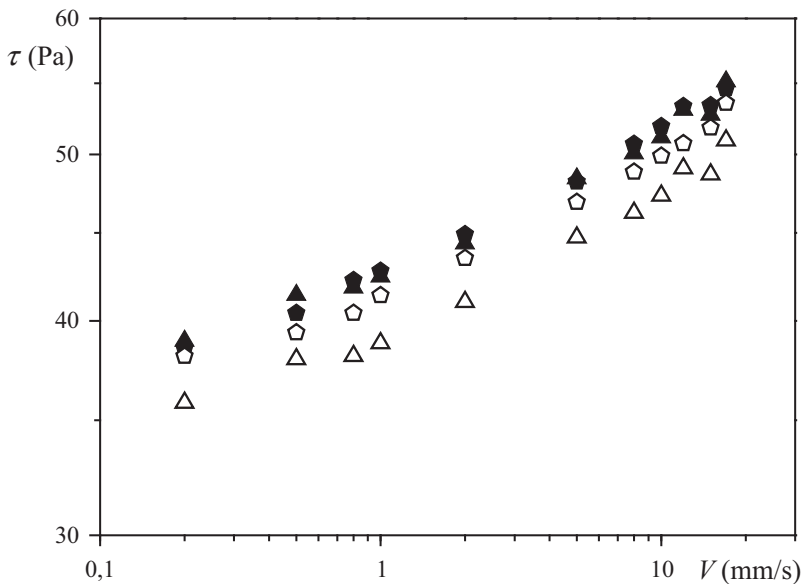

Fig. 13. Viscous shear stress along the plate $\tau$ as a function of the plate velocity $V$ during immersion (full symbols) and withdrawal (open symbols) for $33 \mathrm{~Pa}$, computed with the equation deduced from the force curves (triangles) and from the velocity gradient near the plate (pentagons). 


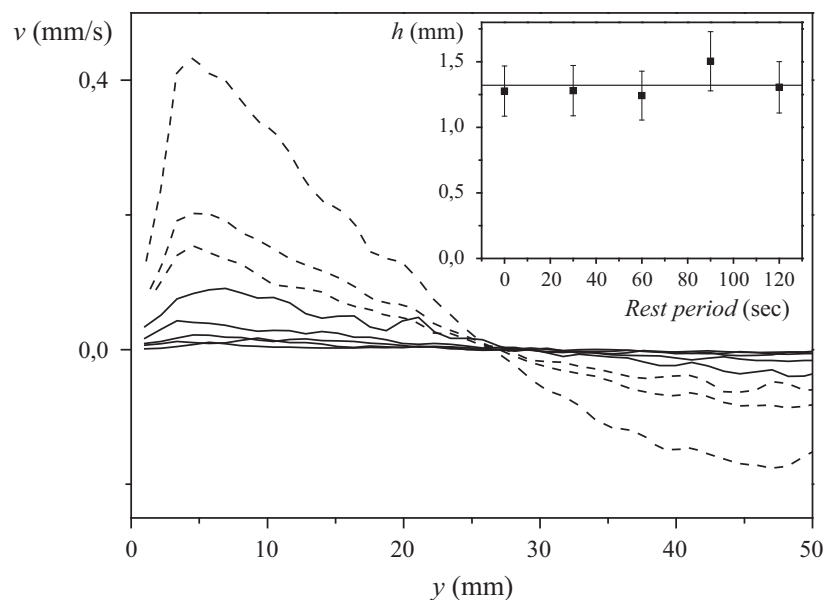

Fig. 14. Velocity profiles (at a depth $4 \mathrm{~cm}$ ) during a rest period after a $200 \mathrm{~mm}$ plate immersion at $V=5 \mathrm{~mm} / \mathrm{s}$ and $\tau_{c}=33 \mathrm{~Pa}$. Successive profiles in time are observed from top to bottom for short distance. The dashed lines correspond to the initial successive velocity profiles (every $2 / 3 \mathrm{~s}$ ), the continuous lines to the next times (every $2 \mathrm{~s}$ ). Inset: coated layer thickness as a function of the duration of the rest period before withdrawal.

$(2 / 3 \mathrm{~s})$. This means that the noise on these data is significant and they must be regarded essentially as providing indications on qualitative trends.

First it is remarkable that the coated layer thickness is independent of the rest period between immersion and withdrawal (see inset of Fig. 14). Since there is likely a strong link between the sheared layer along the plate in the bath and the coated thickness this suggests that the liquid layer along the plate is poorly affected by what can occur during the relaxation stage.

Let us now look at the impact of the time of rest on force measurements. The force versus tip position curves (see inset of Fig. 15) are exactly superimposed with or without rest before immersion, which means that the flow characteristics are independent of the time of rest. This result is consistent with the observation that a rest period does not influence the thickness of the coated layer (see above).

The force versus time curves also makes it possible to appreciate the stress relaxation during the rest period (see Fig. 15). The characteristic relaxation time is of the order of $10 \mathrm{~s}$. This relaxation

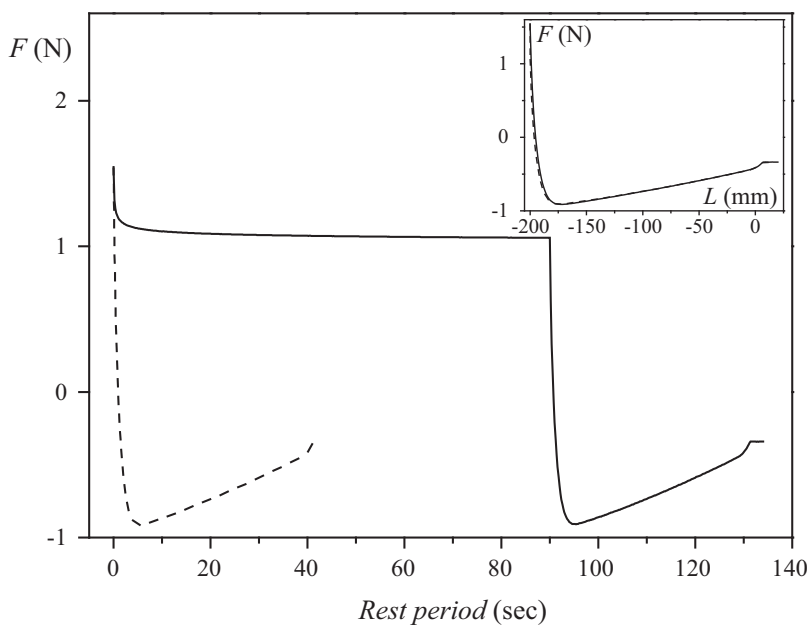

Fig. 15. Force applied on the plate during its withdrawal as a function of time for $\tau_{c}=33 \mathrm{~Pa}$ and $V=5 \mathrm{~mm} / \mathrm{s}$, with no rest period (dash line) and after a rest period of $90 \mathrm{~s}$ (continuous line). Inset: force applied on the plate during its withdrawal as a function of the lower tip depth for $\tau_{c}=33 \mathrm{~Pa}$ and $V=5 \mathrm{~mm} / \mathrm{s}$, with no rest period (dashed line) and after a rest period of $90 \mathrm{~s}$ (continuous line). process also appears in the velocity profiles (see Fig. 14). There is a kind of motion of equilibration in which the fluid close to the plate (below $20 \mathrm{~mm}$ ) moves upwards along the stopped plate while the fluid farther from the plate moves downwards. These motions progressively damp over about $8 \mathrm{~s}$. It is likely that this equilibration is due to the elastic strains in the solid part: when the plate is stopped, the elastic regions relax by shearing the fluid in the region along the plate. Here we can remark that the relaxation time is close to that observed for the force curve after stoppage, which confirms that the same process is at the origin of both effects.

In accordance to [31], with the value of the force plateau during relaxation, it is then possible to deduce the material yield stress using the equation in the viscous force (see Section 3.3).

\section{Conclusion}

The flows generated by the plate immersion and withdrawal are similar: both involve the formation of a liquid layer of uniform thickness along the plate during its displacement, while the rest of the fluid is in its solid state. Then, when withdrawn from the bath, the plate is uniformly coated with a millimetric layer of fluid. This result can be easily generalized to any type of solid object withdrawn from a bath of yield stress fluid: in the absence of wall slip, as no drainage occurs, there is a critical coated thickness at vanishing velocities.

Precise studies of the force curves and of the velocity fields inside the bath give qualitative and quantitative information on the impact of the experimental parameters: the liquid and coated uniform layers both increase with the plate velocity and the fluid yield stress while they are independent of the time of rest before the plate withdrawal and of the overall experiment dimensions. Moreover, the shear stress applied along the plate slowly increases with the plate velocity and significantly with the fluid yield stress.

From an industrial point of view (painting, surface treatment, food coating), these conclusions show that in the studied ranges, the geometrical requirements on the objects and container dimensions are not very restricting providing there is several centimeters between the two plates or the plate and the container wall to coat and the solid and the container. Then, as the coated thickness is mainly governed by the fluid yield stress, a decrease of this quantity is efficient to minimize the volume of fluid coated on the object and the extension of the non uniform coated zones. As the force applied on the plate decreases with the yield stress, its decrease also reduces the energy needed to cover the plate and no time of rest is needed between immersion and withdrawal.

To conclude, in the present paper, we improved several techniques introduced in $[30,31]$ to describe precisely the flow developed along the plate during its withdrawal through the force curves and PIV analysis. Since it has been shown that the coated layer thickness is approximately proportional to the yield stress [12], it could be useful to understand further the link between the flow in the bath and the coated layer in order to have a new practical technique for determining the yield stress. Numerical works might provide interesting information for further understanding of the link between the thickness of the liquid layer and the coated layer.

\section{References}

[1] K.J. Ruschak, Coating flows, Annu. Rev. Fluid Mech. 17.1 (1985) 65-89.

[2] D. Quéré, Fluid coating on a fiber, Annu. Rev, Fluid Mech. 31.1 (1999) 347-384.

[3] J.H. Snoeijer, J. Ziegler, B. Andreotti, M. Fermigier, J. Eggers, Thick films of viscous fluid coating on a plate withdrawn from a liquid reservoir, Phys. Rev. Lett. 100.24 (2008) 244502.

[4] H.C. Mayer, R. Krechetnikov, Landau-Levich flow visualization: revealing the flow topology responsible for the film thickening phenomena (1994-present), Phys. Fluids 24.5 (2012) 052103. 
[5] A. de Ryck, D. Quéré, Fluid coating from a polymer solution, Langmuir 14.7 (1998) 1911-1914.

[6] J. Ashmore, A.Q. Shen, H.P. Kavehpour, H.A. Stone, G.H. McKinley, Coating flows of non-Newtonian fluids: weakly and strongly elastic limits, J. Eng. Math. 60.1 (2008) 17-41.

[7] F. Kamışlı, Free coating of a non-Newtonian liquid onto walls of a vertical and inclined tube, Chem. Eng. Process. 42.7 (2003) 569-581.

[8] C. Gutfinger, J.A. Tallmadge, Films of non-Newtonian fluids adhering to flat plates, AIChE J. 11.3 (1965) 403-413.

[9] P. Hurez, P.A. Tanguy, Finite element analysis of dip coating with Bingham fluids, Polym. Eng. Sci. 30.18 (1990) 1125-1132.

[10] A. Abdelkader, L. Khezzar, E. Mitsoulis, Some experiences with the numerica simulation of Newtonian and Bingham fluids in dip coating, Comput. Fluids 82 (2013) 110-121.

[11] B.V. Derjaguin, S.M. Levi, Film Coating Theory, The Focal Press, London, 1964.

[12] M. Maillard, J. Boujlel, P. Coussot, Solid-solid transition in Landau-Levich flow with soft-jammed systems, Phys. Rev. Lett. 112.6 (2014) 068304.

[13] P. Coussot, Yield stress fluid flows: a review of experimental data, J. NonNewton. Fluid Mech. 211 (2014) 31-49.

[14] R.W. Ansley, T.N. Smith, Motion of spherical particles in a Bingham plastic, AIChE J. 13 (1967) 1193-1196.

[15] A.N. Beris, J.A. Tsamopoulos, R.C. Armstrong, R.A. Brown, Creeping motion of a sphere through a Bingham Plastic, J. Fluid Mech. 158 (1985) 219-244.

[16] J. Blackery, E. Mitsoulis, Creeping motion of a sphere in tubes filled with a Bingham plastic material, J. Non-Newton. Fluid Mech. 70 (1997) 59-77.

[17] M. Beaulne, E. Mitsoulis, Creeping motion of a sphere in tubes filled with Herschel-Bulkley fluids, J. Non-Newton. Fluid Mech. 72 (1997) 55-71.

[18] D.L. Tokpavi, A. Magnin, P. Jay, Very slow flow of Bingham viscoplastic fluid around a circular cylinder, J. Non-Newton. Fluid Mech. 154 (2008) 65-76.

[19] A. Putz, I.A. Frigaard, Creeping flow around particles in a Bingham fluid, J. NonNewton. Fluid Mech. 165 (2010) 263-280.

[20] D.D. Atapattu, R.P. Chhabra, P.H.T. Uhlherr, Creeping sphere motion in Herschel-Bulkley fluids: flow field and drag, J. Non-Newton. Fluid Mech. 59 (1995) 245-265.
[21] L. Jossic, A. Magnin, Drag and stability of objects in a yield stress fluid, AIChE J. 47 (2001) 2666-2672.

[22] O. Merkak, L. Jossic, A. Magnin, Spheres and interactions between spheres moving at very low velocities in a yield stress fluid, J. Non-Newton. Fluid Mech. 133 (2006) 99-108.

[23] H. Tabuteau, P. Coussot, J. de Bruyn, Drag force on a sphere in steady motion through a yield stress fluid, J. Rheol. 51 (2007) 125-137.

[24] D.L. Tokpavi, P. Jay, A. Magnin, L. Jossic, Experimental study of the very slow flow of a yield stress fluid around a circular cylinder, J. Non-Newton. Fluid Mech. 164 (2009) 35-44.

[25] L. Jossic, F. Ahonguio, A. Magnin, Flow of a yield stress fluid perpendicular to a disc, J. Non-Newton. Fluid Mech. 191 (2013) 14-24.

[26] B. Gueslin, L. Talini, Y. Peysson, Sphere settling in an aging yield stress fluid: link between the induced flows and the rheological behavior, Rheol. Acta 48.9 (2009) 961-970.

[27] A.M.V. Putz, T.I. Burghelea, I.A. Frigaard, D.M. Martinez, Settling of an isolated spherical particle in a yield stress shear thinning fluid, Phys. Fluids 20 (2008) 033102.

[28] F. Ahonguio, L. Jossic, A. Magnin, Influence of surface properties on the flow of a yield stress fluid around spheres, J. Non-Newton. Fluid Mech. 206 (2014) 57-70.

[29] B. Dollet, F. Graner, Two-dimensional flow of foam around a circular obstacle: local measurements of elasticity, plasticity and flow, J. Fluid Mech. 585 (2007) 181-211.

[30] J. Boujlel, M. Maillard, A. Lindner, G. Ovarlez, X. Chateau, P. Coussot, Boundary layer in pastes - displacement of a long object through a yield stress fluid, J. Rheol. 56 (2012) 1083-1108.

[31] J. Boujlel, P. Coussot, Measuring yield stress: a new practical and precise technique derived from detailed penetrometry analysis, Rheol. Acta 51 (2012) 867-882.

[32] P. Coussot, L. Tocquer, C. Lanos, G. Ovarlez, Macroscopic vs local rheology of yield stress fluids, J. Non-Newton. Fluid Mech. 158 (2009) 85-90.

[33] P. Coussot, Rheometry of Pastes, Wiley, 2005.

[34] O. Hassager, Negative wake behind bubbles in non-Newtonian liquids, Nature 279 (1979) 402. 